\title{
Fuzzy Adaptive Prescribed Performance Control for a Class of Uncertain Nonlinear Systems with Unknown Dead-Zone Inputs
}

\author{
Wei Xiang, Ning Li, and Yeguo Sun \\ Department of Mathematics, Huainan Normal University, Huainan 232038, China \\ Correspondence should be addressed to Wei Xiang; xiangwei27@126.com
}

Received 13 July 2016; Accepted 16 October 2016; Published 26 March 2017

Academic Editor: Hung-Yuan Chung

Copyright (C) 2017 Wei Xiang et al. This is an open access article distributed under the Creative Commons Attribution License, which permits unrestricted use, distribution, and reproduction in any medium, provided the original work is properly cited.

\begin{abstract}
This paper proposes a fuzzy adaptive prescribed performance control scheme for a class of uncertain chaotic systems with unknown control gains and unknown dead-zone inputs. Firstly, an error transformation is introduced to transform the original constrained system into an equivalent unconstrained one. Then, based on the error transformation technique and the predefined performance technique, a fuzzy adaptive feedback control method is developed. It is shown that all the signals of the resulting closed-loop system are bounded. Finally, an illustrative example is given to demonstrate the effectiveness and usefulness of the proposed technique.
\end{abstract}

\section{Introduction}

As a hard nonlinearity, dead-zone has been found in many industrial processes. For example, dry friction or stiction is a common source of dead-zone nonlinearities in electromechanical systems, and temperature changes on the surface of these components can produce relevant variations of deadzone effects [1]. If dead-zone nonlinearities are neglected [2], they will limit the closed-loop system performance and lead to instability. So, these nonlinearities are particularly harmful. Many researchers employed many methods to improve the performances of control systems with dead-zone inputs. The most common approaches are adaptive schemes [3-6], fuzzy systems [7-14], neural networks [15-18], and sliding mode control [19-22]. In order to compensate the negative effects of the dead-zone nonlinearity, an inverse dead-zone as a method is used. But this method leads to a discontinuous control law and requires instantaneous switching, which in practice cannot be accomplished with mechanical actuators. To overcome this limitation, smooth inverses are adopted in $[23,24]$. Another method was proposed by Lewis et al. [8] and adopted by Wang et al. [25]. In both works, the dead-zone is treated as a combination of a linear and a saturation function. In [4], Boulkroune and M'Saad proposed fuzzy adaptive observer-based approach to deal with the practical projective synchronization problem for a class of chaotic systems involving dead-zone in the control channel. $\mathrm{Wu}$ et al. [5] introduced a smooth inverse of the dead-zone to compensate the effect of the dead-zone in controllers design and proposed an adaptive sliding mode control law to achieve spacecraft attitude tracking problem. By using a four-dimensional energy resource demand supply system, an adaptive neural networks control approach is presented in [17]. The approach not only makes the states of two chaotic systems asymptotic synchronization but also achieved better control performances. In [19], an adaptive fuzzy control scheme is proposed for a class of uncertain multi-input multioutput (MIMO) nonlinear systems with the nonsymmetric control gain matrix and the unknown dead-zone inputs. Boulkroune and M'Saad [20] developed a fuzzy adaptive variable-structure control scheme for a class of uncertain MIMO chaotic systems with both sector nonlinearities and dead-zones. In order to realize the robust compensator, most of the aforementioned control schemes are obtained 
with the restriction that the control gains are known in advance. However, this assumption does not appear to be realistic in a general case [26]. So it is more advisable to take the effects of the unknown control gains and unknown dead-zone inputs into account for uncertain nonlinear systems.

Recently, Bechlioulis and Rovithakis [27] proposed prescribed performance control (PPC) scheme for uncertain nonlinear system. Utilizing a transformation function, the original controlled system is transformed into a new one. If the uniform boundedness of the states of the latter is ensured, we can solve the stability problem for the former. The paper [28] established a control scheme to control unknown pure feedback systems of known high relative degree, exhibiting prescribed performance with respect to trajectory oriented metrics. For nonlinear large-scale systems, Li and Tong [29] employed prescribed performance adaptive fuzzy outputfeedback control method to ensure that all the signals in the closed-loop system are bounded. Meanwhile, Li and Tong [30] proposed an adaptive fuzzy output constrained control design approach for MIMO uncertain stochastic nonlinear systems. Sun and Liu [31] presented a fuzzy adaptive control method for MIMO uncertain chaotic systems, which is capable of guaranteeing the prescribed performance bounds. However, the main limitation in [31] is that the effect of both unknown control gains and unknown dead-zone inputs for uncertain nonlinear systems has not been taken into account.

To the author's best knowledge, there are few literatures dealing with the prescribed performance control problem with unknown dead-zone inputs. Inspired by the works in [32], we investigate the tracking control with guaranteed prescribed performance for uncertain nonlinear systems. Compared with related works, there are three main contributions that are worth to be emphasized:

(1) Compared with the results in [31], the uncertain chaotic system with unknown control gain and unknown dead-zone input is considered.

(2) The prescribed performance function (PPF) is incorporated into the control design.

(3) The proposed control law avoids the chattering phenomenon.

Motivated by the aforementioned works, this paper focuses on the problem of adaptive fuzzy control for a class of uncertain nonlinear systems with unknown control gains and unknown dead-zone inputs. Based on Lyapunov function, it is proved that all the signals of the closed-loop system are bounded and that the tracking error remains an adjustable neighborhood of the origin with the prescribed performance bounds.

The organization of this paper is described as follows. In the next section, system model is derived, and the assumptions are also given. In Section 3, the design of the proposed control strategies is discussed. The simulation results are presented to demonstrate the effectiveness of proposed control scheme in Section 4. Conclusion is presented in Section 5 .

\section{System Descriptions and Problem Formulations}

Consider the following nonlinear system:

$$
\begin{gathered}
\dot{x}_{1}=f_{1}(t, x)+g_{1}(t, x) \phi_{1}\left(u_{1}(t)\right), \\
\dot{x}_{2}=f_{2}(t, x)+g_{2}(t, x) \phi_{2}\left(u_{2}(t)\right), \\
\vdots \\
\dot{x}_{n}=f_{n}(t, x)+g_{n}(t, x) \phi_{n}\left(u_{n}(t)\right),
\end{gathered}
$$

where $x=\left[x_{1}, x_{2}, \ldots, x_{n}\right]^{T}$ is the system state vector which is assumed to be available for measurement. $u=$ $\left[u_{1}, u_{2}, \ldots, u_{n}\right]^{T}$ is the control input vector and $f(t, x)=$ $\left[f_{1}(t, x), f_{2}(t, x), \ldots, f_{n}(t, x)\right]^{T}$ is the unknown continuous nonlinear function, $g(t, x)=\left[g_{1}(t, x), g_{2}(t, x), \ldots, g_{n}(t, x)\right]^{T}$ is unknown control gain, and $\phi_{i}\left(u_{i}(t)\right)$ is the output of the dead-zone nonlinearity, $i=1,2, \ldots, n$.

The dead-zone characteristic can be described as follows:

$$
\begin{aligned}
& \phi_{i}\left(u_{i}(t)\right) \\
& = \begin{cases}m_{i}\left(u_{i}(t)-b_{1 i}\right), & \text { for } u_{i}(t) \geq b_{1 i} \\
0, & \text { for }-b_{2 i}<u_{i}(t)<b_{1 i} \\
m_{i}\left(u_{i}(t)+b_{2 i}\right), & \text { for } u_{i}(t) \leq-b_{2 i},\end{cases}
\end{aligned}
$$

where $m_{i}$ stand for the right and the left slope, $b_{1 i}$ and $b_{2 i}$ represent the breakpoints of the input nonlinearity, and the following assumption is given.

Assumption 1. The dead-zone parameters: $m_{i}>0, b_{1 i}$ and $b_{2 i}$ are unknown bounded constants.

Obviously, the output of the dead-zone can be rewritten as

$$
\phi_{i}\left(u_{i}(t)\right)=m_{i} u_{i}(t)+d_{i}\left(u_{i}(t)\right),
$$

where $d_{i}\left(u_{i}(t)\right)$ can be calculated as

$$
d_{i}\left(u_{i}(t)\right)= \begin{cases}-m_{i} b_{1 i}, & \text { for } u_{i}(t) \geq b_{1 i} \\ -m_{i} u_{i}(t), & \text { for }-b_{2 i}<u_{i}(t)<b_{1 i} \\ m_{i} b_{2 i}, & \text { for } u_{i}(t) \leq-b_{2 i} .\end{cases}
$$

From Assumption 1, there exists an unknown positive constant $d_{i}^{*}$ such as $\left|d_{i}\left(u_{i}(t)\right)\right| \leq d_{i}^{*}, i=1,2, \ldots, n$.

Now, let $\Phi(u)=\left[\phi_{1}\left(u_{1}(t)\right), \phi_{2}\left(u_{2}(t)\right), \ldots, \phi_{n}\left(u_{n}(t)\right)\right]^{T}$, $D(u)=\left[d_{1}\left(u_{1}(t)\right), d_{2}\left(u_{2}(t)\right), \ldots, d_{n}\left(u_{n}(t)\right)\right]^{T}$, and $M=$ $\operatorname{diag}\left(m_{1}, m_{2}, \ldots, m_{n}\right)$. From (1) and (3), we have

$$
\begin{aligned}
& \dot{x}=f(t, x)+\operatorname{diag}(g(t, x)) \Phi(u), \\
& \Phi(u)=M u+D(u) .
\end{aligned}
$$

So, we can obtain

$$
\begin{aligned}
\dot{x}= & f(t, x)+\operatorname{diag}(g(t, x)) M u \\
& +\operatorname{diag}(g(t, x)) D(u) .
\end{aligned}
$$


The objective of this paper is to construct a controller for system (1) such that the system state $x$ tracks the reference signal $x_{d} \in R^{n}$ and all the signals in the closed-loop system remain bounded.

To meet the objective, we make the following reasonable assumptions.

Assumption 2. $f(t, x)$ and $\operatorname{diag}(g(t, x))$ are unknown but bounded.

Assumption 3. The matrix $\operatorname{diag}(g(t, x))$ is nonsingular.

Assumption 4. The desired trajectory $x_{i d}$ is a known bounded differentiable function, $i=1,2, \ldots, n$.

Remark 5. In [19], $g_{i}(t, x)$ is assumed as $g_{i}(t, x) \geq t_{i}$, and $\iota_{i}$ is positive constant, $i=1,2, \ldots, n$. In this paper, we relax this condition and only suppose $\operatorname{diag}(g(t, x))$ is an unknown nonsingular matrix.

Let $x_{d}=\left[x_{1 d}, x_{2 d}, \ldots, x_{n d}\right]^{T}$, and the tracking error $e=$ $x-x_{d}=\left[e_{1}, e_{2}, \ldots, e_{n}\right]^{T}$. So we can obtain the following error dynamical system:

$$
\begin{aligned}
\dot{e}= & f(t, x)+\operatorname{diag}(g(t, x)) M u+\operatorname{diag}(g(t, x)) D(u) \\
& -\dot{x}_{d} .
\end{aligned}
$$

Usually, we employ sliding mode control scheme [18] to stabilize the error system (7). Firstly, a sliding mode surface is designed as follows:

$$
\sigma=e+\int_{0}^{t} K e(\tau) \mathrm{d} \tau,
$$

where $K=\operatorname{diag}\left(k_{1}, k_{2}, \ldots, k_{n}\right), k_{i}>0, i=1,2, \ldots, n$. Differentiating $\sigma$ with time yields

$$
\begin{aligned}
\dot{\sigma}= & f(t, x)+\operatorname{diag}(g(t, x)) M u+\operatorname{diag}(g(t, x)) D(u) \\
& -\dot{x}_{d}+K e .
\end{aligned}
$$

If the nonlinear functions $f(t, x)$ and $\operatorname{diag}(g(t, x))$ and the parameters $m_{i}$ and $d_{i}^{*}$ are all known, we employ the control law

$$
\begin{aligned}
u= & -(\operatorname{diag}(g(t, x)) M)^{-1} \\
& \cdot\left(f(t, x)-\dot{x}_{d}+K e+K \sigma+\bar{u}_{s}\right),
\end{aligned}
$$

where $\bar{u}_{s}=\left[\left|g_{1}(t, x)\right| d_{1}^{*} \cdot \operatorname{sign}\left(\sigma_{1}\right),\left|g_{2}(t, x)\right| d_{2}^{*} \cdot \operatorname{sign}\left(\sigma_{2}\right)\right.$, $\left.\ldots,\left|g_{n}(t, x)\right| d_{n}^{*} \cdot \operatorname{sign}\left(\sigma_{n}\right)\right]^{T}$.

Consider the Lyapunov function $V_{0}=(1 / 2) \sigma^{T} \sigma$. Using (10) yields

$$
\begin{aligned}
\dot{V}_{0}= & \sigma^{T} \dot{\sigma} \\
= & -\sigma^{T} K \sigma \\
& -\sum_{i=1}^{n}\left(\left|\sigma_{i}\right|\left|g_{i}(t, x)\right| d_{i}^{*}-\sigma_{i} g_{i}(t, x) d_{i}\left(u_{i}\right)\right) .
\end{aligned}
$$

One obtains $\dot{V}_{0} \leq-\sigma^{T} K \sigma \leq 0$. We can conclude that $\sigma \rightarrow 0$ as $t \rightarrow \infty$. However, the control law (10) is implemented in cases where $f(t, x), \operatorname{diag}(g(t, x)), m_{i}$, and $d_{i}^{*}$ are all known. And $u_{s}$ may cause chattering phenomena. Meanwhile, the inverse of $\operatorname{diag}(g(t, x)) M$ cannot be calculated easily.

In order to overcome these limitations, we adopt the fuzzy adaptive prescribed performance control scheme to ensure that all the signals are bounded in probability and the system state $x(t)$ can track the given reference $x_{d}$ with the given prescribed performance bounds.

2.1. Prescribed Performance. According to $[14,15]$, the prescribed performance is achieved by ensuring that tracking error $e=\left[e_{1}, e_{2}, \ldots, e_{n}\right]^{T}$ evolves strictly within predefined decaying bounds as follows:

$$
\begin{aligned}
-\delta_{i \min } \mu_{i}(t)<e_{i}(t)<\delta_{i \max } \mu_{i}(t), & \\
t & \geq 0, i=1,2, \ldots, n,
\end{aligned}
$$

where $\delta_{i \min }$ and $\delta_{i \max }$ are design constants and the performance functions $\mu_{i}(t)$ are bounded and strictly positive decreasing smooth functions and $\lim _{t \rightarrow \infty} \mu_{i}(t)=\mu_{i \infty}>0$. Choosing the performance function $\mu_{i}(t)$ and the constants $\delta_{i \min }$ and $\delta_{i \max }$ appropriately determines the performance bounds of the error $e_{i}, i=1,2, \ldots, n$.

To represent (11) by an equality form, we employ an error transformation as

$$
e_{i}(t)=\mu_{i}(t) s_{i}\left(z_{i}\right), \quad i=1,2, \ldots, n,
$$

where $z_{i}$ is the transformed error, and $s_{i}(\cdot)$ is smooth, strictly increasing function, satisfying the following condition:

$$
\begin{aligned}
-\delta_{i \min } & \leq s_{i}\left(z_{i}\right) \leq \delta_{i \max }, \\
\lim _{z_{i} \rightarrow-\infty} s_{i}\left(z_{i}\right) & =-\delta_{i \min }, \\
\lim _{z_{i} \rightarrow+\infty} s_{i}\left(z_{i}\right) & =\delta_{i \max } .
\end{aligned}
$$

Note that $s_{i}\left(z_{i}\right)$ are strictly increasing functions; we have

$$
z_{i}=s_{i}^{-1}\left(\frac{e_{i}(t)}{\mu_{i}(t)}\right), \quad i=1,2, \ldots, n .
$$

Differentiating (15) with respect to time yields

$$
\begin{aligned}
\dot{z}_{i} & =\frac{\partial s_{i}^{-1}}{\partial\left(e_{i}(t) / \mu_{i}(t)\right)} \frac{1}{\mu_{i}(t)}\left[f_{i}(t, x)+g_{i}(t, x) m_{i} u_{i}\right. \\
& \left.+g_{i}(t, x) d_{i}\left(u_{i}\right)-\dot{x}_{d i}-\frac{e_{i}(t) \dot{\mu}_{i}(t)}{\mu_{i}(t)}\right] .
\end{aligned}
$$

Let

$$
\begin{aligned}
r_{i} & =\frac{\partial s_{i}^{-1}}{\partial\left(e_{i}(t) / \mu_{i}(t)\right)} \frac{1}{\mu_{i}(t)}>0, \\
h_{i} & =-\dot{x}_{d i}-\frac{e_{i}(t) \dot{\mu}_{i}(t)}{\mu_{i}(t)} .
\end{aligned}
$$


Then (16) can be rewritten as

$$
\begin{array}{r}
\dot{z}_{i} \\
=r_{i}\left[f_{i}(t, x)+g_{i}(t, x) m_{i} u_{i}+g_{i}(t, x) d_{i}\left(u_{i}\right)+h_{i}\right], \\
i=1,2, \ldots, n .
\end{array}
$$

Let $z=\left[z_{1}, z_{2}, \ldots, z_{n}\right]^{T}, H=\left[h_{1}, h_{2}, \ldots, h_{n}\right]^{T}$; then (18) can be written into the following form:

$$
\begin{aligned}
\dot{z}= & \operatorname{diag}(r)[f(t, x)+\operatorname{diag}(g(t, x)) M u \\
& +\operatorname{diag}(g(t, x)) D(u)+H]
\end{aligned}
$$

where $\operatorname{diag}(r)=\operatorname{diag}\left(r_{1}, r_{2}, \ldots, r_{n}\right)$.

Remark 6. Usually, we choose $s_{i}\left(z_{i}\right)=\tanh \left(z_{i}\right)=\left(\delta_{i \max } e^{z_{i}}-\right.$ $\left.\delta_{i \min } e^{-z_{i}}\right) /\left(e^{z_{i}}+e^{-z_{i}}\right)$. So, we can calculate that $r_{i}=\left(1 /\left(\lambda_{i}+\right.\right.$ $\left.\left.\delta_{i \text { min }}\right)+1 /\left(\delta_{i \max }-\lambda_{i}\right)\right) / 2 \mu>0, \lambda_{i}=e_{i}(t) / \mu_{i}(t)$.
If $f_{i}(t, x), g_{i}(t, x), m_{i}$, and $d_{i}^{*}$ are all known, the following controller

$$
u=-(\operatorname{diag}(g(t, x)) M)^{-1}\left[f(t, x)+H+K z+u_{s}\right],
$$

where $u_{s}=\left[\operatorname{sign}\left(z_{1}\right)\left|g_{1}(t, x)\right| d_{1}^{*}, \operatorname{sign}\left(z_{2}\right)\left|g_{2}(t, x)\right| d_{2}^{*}, \ldots\right.$, $\left.\operatorname{sign}\left(z_{n}\right)\left|g_{n}(t, x)\right| d_{n}^{*}\right]^{T}$, and $K=\operatorname{diag}\left(k_{1}, k_{2}, \ldots, k_{n}\right)$ with $k_{i}>$ $0(i=1,2, \ldots, n)$, can meet the control objective. Indeed, consider the Lyapunov function $V_{1}=(1 / 2) z^{T} z$. Using (19) and (20) yields $\dot{V}_{1}=z^{T} \dot{z} \leq 0$. According to the above inequality, $V_{1}$ is always negative, which implies that $z_{i} \in L_{\infty}$. Then, according to the properties of function $s_{i}\left(z_{i}\right)$, we know that $-\delta_{i \text { min }}<s_{i}\left(z_{i}\right)<\delta_{i \max }$, which implies $-\delta_{i \min } \mu_{i}(t)<$ $e_{i}(t)<\delta_{i \max } \mu_{i}(t)$. Then, one can conclude that tracking control of system (2) is achieved.

However, the term $u_{s}$ is discontinuous, which may cause chattering phenomena, and inverse matrix $(\operatorname{diag}(g(t$, $x)) M)^{-1}$ cannot be calculated easily. So, motivated by $[11,12]$, we modify controller (20) as follows:

$$
u=-\frac{z\left[z^{T} \operatorname{diag}(r) f(t, x)+z^{T} \operatorname{diag}(r) H+z^{T} \operatorname{diag}(r) K z+\bar{z}^{T} \operatorname{diag}(r) \bar{u}_{s}\right]}{z^{T} \operatorname{diag}(r) \operatorname{diag}(g(t, x)) M z},
$$

where $\bar{z}=\left[\left|z_{1}\right|,\left|z_{2}\right|, \ldots,\left|z_{n}\right|\right]^{T}, \bar{u}_{s}=\left[\left|g_{1}(t, x)\right| d_{1}^{*}, \mid g_{2}(t\right.$, $\left.x)\left|d_{2}^{*}, \ldots,\right| g_{n}(t, x) \mid d_{n}^{*}\right]^{T}$. The control objective can also be realized.

Remark 7. In controller (21), we do not need to calculate the matrix $(\operatorname{diag}(g(t, x)) M)^{-1}$.

Note that $f(t, x), \operatorname{diag}(g(t, x)), M$, and $\bar{u}_{s}$ are unknown in (21); we need to use fuzzy logic system to approximate the nonlinear unknown functions.

2.2. Fuzzy Logic Systems. The basic configuration of a fuzzy logic system consists of a fuzzifier, some fuzzy IF-THEN rules, a fuzzy inference engine, and a defuzzifier. The fuzzy inference engine uses the fuzzy IF-THEN rules to perform a mapping from an input vector $x=\left[x_{1}, x_{2}, \ldots, x_{n}\right]^{T} \in R^{n}$ to an output $\alpha(x) \in R$. The $i$ th fuzzy rule is written as

Rule $i$ : if $x_{1}$ is $F_{1}^{i}$ and $\ldots$ and $x_{n}$ is $F_{n}^{i}$ then $\alpha(x)$ is $\alpha_{i}$, where $F_{1}^{i}, F_{2}^{i}, \ldots$, and $F_{n}^{i}$ are fuzzy sets and $\alpha_{i}$ is the fuzzy singleton for the output in the $i$ th rule. By using the singleton fuzzifier, product inference, and the center-average defuzzifier, the output of the fuzzy system can be expressed as follows:

$$
\alpha(x)=\frac{\sum_{j=1}^{N} \alpha_{j} \prod_{i=1}^{n} \mu_{F_{i}^{j}}\left(x_{i}\right)}{\sum_{j=1}^{N}\left[\prod_{i=1}^{n} \mu_{F_{i}^{j}}\left(x_{i}\right)\right]}=\theta^{T} \psi(x),
$$

where $\mu_{F_{i}^{j}}\left(x_{i}\right)$ is the degree of membership of $x_{i}$ to $F_{i}^{j}, N$ is the number of fuzzy rules, $\theta=\left[\alpha_{1}, \ldots, \alpha_{N}\right]^{T}$ is the adjustable parameter vector, and $\psi(x)=\left[p_{1}(x), p_{2}(x), \ldots, p_{N}(x)\right]^{T}$, where

$$
p_{j}(x)=\frac{\prod_{i=1}^{n} \mu_{F_{i}^{j}}\left(x_{i}\right)}{\sum_{j=1}^{N}\left[\prod_{i=1}^{n} \mu_{F_{i}^{j}}\left(x_{i}\right)\right]}
$$

is the fuzzy basis function. It is assumed that fuzzy basis functions are selected so that there is always at least one active rule.

\section{Main Results}

Let $\operatorname{diag}(\bar{g}(t, x))=\operatorname{diag}(g(t, x)) M=\operatorname{diag}\left(g_{1}(t, x) m_{1}, g_{2}(t\right.$, $\left.x) m_{2}, \ldots, g_{n}(t, x) m_{n}\right)$. By applying the introduced fuzzy systems, approximation of functions $f_{i}(t, x), g_{i}(t, x) m_{i}$, and $\left|g_{i}(t, x)\right| d_{i}^{*}\left(u_{i}\right)$ can be expressed as follows:

$$
\begin{aligned}
\widehat{f}_{i}\left(x, \theta_{f_{i}}\right) & =\theta_{f_{i}}^{T} \psi_{f_{i}}(x), \\
\widehat{\bar{g}}_{i}\left(x, \theta_{g_{i}}\right) & =\theta_{g_{i}}^{T} \psi_{g_{i}}(x), \\
\widehat{g}_{i}\left(x, \theta_{\left|g_{i}\right|}\right) & =\theta_{\left|g_{i}\right|}^{T} \psi_{\left|g_{i}\right|}(x), \\
& i=1,2, \ldots, n .
\end{aligned}
$$

Optimal parameters $\theta_{f_{i}}^{*}, \theta_{g_{i}}^{*}$, and $\theta_{\left|g_{i}\right|}^{*}$ can be defined such that

$$
\theta_{f_{i}}^{*}=\underset{\theta_{f_{i}}}{\operatorname{argmin}}\left[\sup \left|f_{i}(t, x)-\widehat{f}_{i}\left(x, \theta_{f_{i}}\right)\right|\right],
$$




$$
\begin{aligned}
& \theta_{g_{i}}^{*}=\underset{\theta_{g_{i}}}{\operatorname{argmin}}\left[\sup \left|g_{i}(t, x) m_{i}-\hat{\bar{g}}_{i}\left(x, \theta_{g_{i}}\right)\right|\right], \\
& \theta_{\left|g_{i}\right|}^{*}=\underset{\theta_{\left|g_{i}\right|}}{\operatorname{argmin}}\left[\sup || g_{i}(t, x)\left|d_{i}^{*}-\hat{g}_{i}\left(x, \theta_{\left|g_{i}\right|}\right)\right|\right] \text {, } \\
& i=1,2, \ldots, n \text {. } \\
& \varepsilon_{g_{i}}(x)=g_{i}(t, x) m_{i}-g_{i}\left(x, \theta_{g_{i}}^{*}\right), \\
& \varepsilon_{\left|g_{i}\right|}(x)=\left|g_{i}(t, x)\right| d_{i}^{*}-g_{i}\left(x, \theta_{\left|g_{i}\right|}^{*}\right), \\
& i=1,2, \ldots, n .
\end{aligned}
$$

Define the parameter estimation errors and the fuzzy approximation errors as follows:

$$
\begin{aligned}
\widetilde{\theta}_{f_{i}} & =\theta_{f_{i}}^{*}-\theta_{f_{i}}, \\
\widetilde{\theta}_{g_{i}} & =\theta_{g_{i}}^{*}-\theta_{g_{i}}, \\
\widetilde{\theta}_{\left|g_{i}\right|} & =\theta_{\left|g_{i}\right|}^{*}-\theta_{\left|g_{i}\right|}, \\
\varepsilon_{f_{i}}(x) & =f_{i}(t, x)-f_{i}\left(x, \theta_{f_{i}}^{*}\right),
\end{aligned}
$$

Assumption 8. $\varepsilon_{f_{i}}(x), \varepsilon_{g_{i}}(x)$, and $\varepsilon_{\left|g_{i}\right|}(x)$ are all bounded, respectively.

Denote $\hat{f}\left(x, \theta_{f}^{*}\right)=\left[\widehat{f}_{1}\left(x, \theta_{f_{1}}^{*}\right), \widehat{f}_{2}\left(x, \theta_{f_{2}}^{*}\right), \ldots, \widehat{f}_{n}\left(x, \theta_{f_{n}}^{*}\right)\right]^{T}$, $\operatorname{diag}\left(\hat{\bar{g}}\left(x, \theta_{g}^{*}\right)\right)=\operatorname{diag}\left(\hat{\bar{g}}_{1}\left(x, \theta_{g_{1}}^{*}\right), \hat{\bar{g}}_{2}\left(x, \theta_{g_{2}}^{*}\right), \ldots, \hat{\bar{g}}_{n}\left(x, \theta_{g_{n}}^{*}\right)\right)$, and $\hat{\bar{u}}_{s}^{*}=\left[\hat{g}_{1}\left(x, \theta_{\left|g_{1}\right|}^{*}\right), \hat{g}_{2}\left(x, \theta_{\left|g_{2}\right|}^{*}\right), \ldots, \hat{g}_{n}\left(x, \theta_{\left|g_{n}\right|}^{*}\right)\right]^{T}$.

The controller can be constructed as

$$
u=z u_{0}
$$

where

$$
u_{0}=\frac{z^{T} \operatorname{diag}(r) \hat{f}\left(x, \theta_{f}\right)+z^{T} \operatorname{diag}(r) H+z^{T} \operatorname{diag}(r) K z+\bar{z}^{T} \operatorname{diag}(r) \hat{\bar{u}}_{s}+u_{r}}{-z^{T} \operatorname{diag}(r) \operatorname{diag}\left(\hat{\bar{g}}\left(x, \theta_{g}\right)\right) z+\mu z^{T} \operatorname{diag}(r) z+u_{r}^{2}},
$$

where $\mu=\left\|\operatorname{diag}\left(\hat{\bar{g}}\left(x, \theta_{g}\right)\right)\right\|+\epsilon, \epsilon$ is a small positive constant, $u_{r}$ is a compensated controller, which will be designed later, and $K=\operatorname{diag}\left(k_{1}, k_{2}, \ldots, k_{n}\right), k_{i}>0, i=1,2, \ldots, n$.

Substituting (27) and (28) into $z^{T} \dot{z}$ yields

$$
\begin{aligned}
z^{T} \dot{z}=- & z^{T} \operatorname{diag}(r) K z+z^{T} \operatorname{diag}(r) \\
& \cdot\left(f(t, x)-\widehat{f}\left(x, \theta_{f}\right)\right)+z^{T} \operatorname{diag}(r) \\
& \cdot\left(\operatorname{diag}(\bar{g}(t, x))-\operatorname{diag}\left(\hat{\bar{g}}\left(x, \theta_{g}\right)\right)\right) z u_{0} \\
+ & \mu z^{T} \operatorname{diag}(r) z u_{0}+u_{r}^{2} u_{0}-u_{r} \\
+ & z^{T} \operatorname{diag}(r) \operatorname{diag}(g(t, x)) D(u)-\bar{z}^{T} \operatorname{diag}(r) \\
& \cdot \hat{\bar{u}}_{s} .
\end{aligned}
$$

Note that $z^{T} \operatorname{diag}(r) \operatorname{diag}(g(t, x)) D(u) \leq \bar{z}^{T} \operatorname{diag}(r) \bar{u}_{s}$. So, (21) can be written as follows:

$$
\begin{aligned}
z^{T} \dot{z}= & -\sum_{i=1}^{n} r_{i} k_{i} z_{i}^{2}+\sum_{i=1}^{n} r_{i} z_{i} \widetilde{\theta}_{f_{i}}^{T} \psi_{f_{i}}(x) \\
& +\sum_{i=1}^{n} r_{i} z_{i}^{2} u_{0} \widetilde{\theta}_{g_{i}}^{T} \psi_{f_{i}}(x)+\sum_{i=1}^{n} r_{i}\left|z_{i}\right| \widetilde{\theta}_{\left|g_{i}\right|}^{T} \psi_{\left|g_{i}\right|}(x) \\
& +\sum_{i=1}^{n} r_{i} z_{i}\left(g_{i}(t, x) d_{i}\left(u_{i}\right)-\left|g_{i}(t, x)\right| d_{i}^{*}\right)-u_{r} \\
& +u_{0} u_{r}^{2}+\Lambda,
\end{aligned}
$$

where $\Lambda=\sum_{i=1}^{n} r_{i} z_{i} \varepsilon_{f_{i}}+\sum_{i=1}^{n} r_{i} z_{i}^{2} u_{0} \varepsilon_{g_{i}}+\sum_{i=1}^{n} r_{i}\left|z_{i}\right| \varepsilon_{\left|g_{i}\right|}+$ $\sum_{i=1}^{n} \mu r_{i} z_{i}^{2} u_{0}$.
According to Assumption 8, there exist unknown positive constants $\varepsilon_{f}, \varepsilon_{g}$, and $\varepsilon_{|g|}$ such that

$$
\begin{gathered}
\max _{i}\left\{\left|\varepsilon_{f_{i}}\right|\right\} \leq \varepsilon_{f}, \\
\max _{i}\left\{\left|\varepsilon_{g_{i}}\right|\right\} \leq \varepsilon_{g}, \\
\max _{i}\left\{\left|\varepsilon_{\left|g_{i}\right|}\right|\right\} \leq \varepsilon_{|g|} .
\end{gathered}
$$

So, we have

$$
\Lambda \leq\left(\varepsilon_{f}+\varepsilon_{|g|}\right) \sum_{i=1}^{n} r_{i}\left|z_{i}\right|+\left(\varepsilon_{g}+\mu\right) \sum_{i=1}^{n} r_{i} z_{i}^{2}\left|u_{0}\right| .
$$

Let $\varepsilon_{f}+\varepsilon_{|g|}=\varepsilon_{1}, \varepsilon_{g}=\varepsilon_{2}$. To generate the approximations $f(t, x), \operatorname{diag}(\bar{g}(t, x)), \bar{u}_{s}, \varepsilon_{1}$, and $\varepsilon_{2}$ online, we choose the following adaptation laws:

$$
\begin{aligned}
& \dot{\theta}_{f_{i}}=\kappa_{f_{i}} r_{i} z_{i} \psi_{f_{i}}(x), \\
& \dot{\theta}_{g_{i}}=\kappa_{g_{i}} r_{i} z_{i}^{2} u_{0} \psi_{g_{i}}(x), \\
& \dot{\theta}_{\left|g_{i}\right|}=\kappa_{\left|g_{i}\right|} r_{i}\left|z_{i}\right| \psi_{\left|g_{i}\right|}(x), \\
& \dot{\bar{\varepsilon}}_{1}=\kappa_{\varepsilon_{1}} \sum_{i=1}^{n} r_{i}\left|z_{i}\right| \\
& \dot{\bar{\varepsilon}}_{2}=\kappa_{\varepsilon_{2}} \sum_{i=1}^{n} r_{i} z_{i}^{2}\left|u_{0}\right|
\end{aligned}
$$


where $\widehat{\varepsilon}_{1}, \widehat{\varepsilon}_{2}$ are the estimates of $\varepsilon_{1}$ and $\varepsilon_{2}$, respectively. $\kappa_{f_{i}}, \kappa_{g_{i}}, \kappa_{\left|g_{i}\right|}, \kappa_{\varepsilon_{1}}$, and $\kappa_{\varepsilon_{2}}$ are positive constants, $i=1,2, \ldots, n$. And $u_{r}$ is designed as

$$
\begin{aligned}
& \dot{u}_{r}=-u_{0} u_{r}+1-\frac{\Pi u_{r}}{u_{r}^{2}+v^{2}}, \\
& \dot{v}=-\frac{\Pi v}{u_{r}^{2}+v^{2}},
\end{aligned}
$$

where $\Pi=\widehat{\varepsilon}_{1} \sum_{i=1}^{n} r_{i}\left|z_{i}\right|+\left(\widehat{\varepsilon}_{2}+\mu\right) \sum_{i=1}^{n} r_{i} z_{i}^{2}\left|u_{0}\right|$.

So, we obtain the following theorem.

Theorem 9 (consider system (19)). Suppose that Assumptions 1-8 are satisfied. Then controller (27) with the adaptation law given by (33) can guarantee all signals in the closed-loop system are bounded in probability, and the tracking error $e(t)$ remains in a neighborhood of the origin within the prescribed performance bounds for all $t \geq 0$.

Proof. Consider a Lyapunov function as $V=V_{2}+V_{3}$, where

$$
\begin{aligned}
V_{2} & =\frac{1}{2}\left\{z^{T} z+\sum_{i=1}^{n} \frac{1}{\kappa_{f_{i}}} \widetilde{\theta}_{f_{i}}^{T} \widetilde{\theta}_{f_{i}}+\sum_{i=1}^{n} \frac{1}{\kappa_{g_{i}}} \widetilde{\theta}_{g_{i}}^{T} \widetilde{\theta}_{g_{i}}\right. \\
& \left.+\sum_{i=1}^{n} \frac{1}{\kappa_{\left|g_{i}\right|}} \widetilde{\theta}_{\left|g_{i}\right|}^{T} \widetilde{\theta}_{\left|g_{i}\right|}\right\}, \\
V_{3} & =\frac{1}{2}\left\{u_{r}^{2}+\nu^{2}+\frac{1}{\kappa_{\varepsilon_{1}}} \widetilde{\varepsilon}_{1}^{2}+\frac{1}{\kappa_{\varepsilon_{2}}} \widetilde{\varepsilon}_{2}^{2}\right\},
\end{aligned}
$$

where $\widetilde{\varepsilon}_{1}=\varepsilon_{1}-\widehat{\varepsilon}_{1}, \widetilde{\varepsilon}_{2}=\varepsilon_{2}-\widehat{\varepsilon}_{2}$.

The time derivative of $V_{2}$ is given by

$$
\begin{aligned}
\dot{V}_{2}= & z^{T} \dot{z} \\
& -\sum_{i=1}^{n}\left\{\frac{1}{\kappa_{f_{i}}} \widetilde{\theta}_{f_{i}}^{T} \dot{\theta}_{f_{i}}+\frac{1}{\kappa_{g_{i}}} \widetilde{\theta}_{g_{i}}^{T} \dot{\theta}_{g_{i}}+\frac{1}{\kappa_{\left|g_{i}\right|}} \widetilde{\theta}_{\left|g_{i}\right|}^{T} \dot{\theta}_{\left|g_{i}\right|}\right\} .
\end{aligned}
$$

Substituting (33) into (36), we have

$$
\begin{aligned}
\dot{V}_{2}= & -\sum_{i=1}^{n} r_{i} k_{i} z_{i}^{2} \\
& +\sum_{i=1}^{n} r_{i} z_{i}\left(g_{i}(t, x) d_{i}\left(u_{i}\right)-\left|g_{i}(t, x)\right| d_{i}^{*}\right)-u_{r} \\
& +u_{0} u_{r}^{2}+\Lambda \leq-\sum_{i=1}^{n} r_{i} k_{i} z_{i}^{2}-u_{r}+u_{0} u_{r}^{2}+\Lambda .
\end{aligned}
$$

Notice that

$$
\begin{aligned}
\Lambda & \leq \varepsilon_{1} \sum_{i=1}^{n} r_{i}\left|z_{i}\right|+\left(\varepsilon_{2}+\mu\right) \sum_{i=1}^{n} r_{i} z_{i}^{2}\left|u_{0}\right| \\
& =\widetilde{\varepsilon}_{1} \sum_{i=1}^{n} r_{i}\left|z_{i}\right|+\widetilde{\varepsilon}_{2} \sum_{i=1}^{n} r_{i} z_{i}^{2}\left|u_{0}\right|+\Pi .
\end{aligned}
$$

Substituting (38) into (37), one can obtain

$$
\begin{aligned}
\dot{V}_{2} \leq & -\sum_{i=1}^{n} r_{i} k_{i} z_{i}^{2}-u_{r}+u_{0} u_{r}^{2}+\widetilde{\varepsilon}_{1} \sum_{i=1}^{n} r_{i}\left|z_{i}\right| \\
& +\widetilde{\varepsilon}_{2} \sum_{i=1}^{n} r_{i} z_{i}^{2}\left|u_{0}\right|+\Pi .
\end{aligned}
$$

The time derivative of $V_{3}$ is

$$
\dot{V}_{3}=u_{r} \dot{u}_{r}+\nu \dot{v}-\frac{1}{\kappa_{\varepsilon_{1}}} \widetilde{\varepsilon}_{1} \dot{\vec{\varepsilon}}_{1}-\frac{1}{\kappa_{\varepsilon_{2}}} \widetilde{\varepsilon}_{2} \dot{\vec{\varepsilon}}_{2} .
$$

Substituting (34) into (40), one gets

$$
\dot{V}_{3}=-u_{r}^{2} u_{0}+u_{r}-\widetilde{\varepsilon}_{1} \sum_{i=1}^{n} r_{i}\left|z_{i}\right|-\widetilde{\varepsilon}_{2} \sum_{i=1}^{n} r_{i} z_{i}^{2}\left|u_{0}\right|-\Pi .
$$

Combining (40) and (41) gives

$$
\dot{V}(t) \leq-\sum_{i=1}^{n} r_{i} k_{i} z_{i}^{2}
$$

Therefore, according to Lyapunov theorem, $V(t)$ is always negative, so, $V(t)$ is uniformly ultimately bounded; thus, the transformed error $z_{i}$ is bounded; that is, $z_{i} \in L_{\infty}$. Then, according to the properties of function $s_{i}\left(z_{i}\right)$, we know that $-\delta_{i \text { min }}<s_{i}\left(z_{i}\right)<\delta_{i \max }$. Then, one can conclude that tracking control of system (7) with prescribed error performance (4) is achieved. This completes the proof.

Remark 10. Compared with the results in [15], the unknown dead-zone inputs are considered in the paper. Meanwhile, the control law (27) can avoid the singular problem.

\section{Numerical Simulations}

In this section, the Genesio chaotic system [33] is also used to illustrate the effectiveness of the proposed control scheme. The uncertain Genesio chaotic system is described:

$$
\begin{aligned}
\dot{x}_{1}= & \underbrace{x_{2}+d_{1}(t, x)}_{f_{1}(t, x)}+g_{1}(t, x) \phi_{1}\left(u_{1}\right)(t), \\
\dot{x}_{2}= & \underbrace{x_{3}+d_{2}(t, x)}_{f_{2}(t, x)}+g_{2}(t, x) \phi_{2}\left(u_{2}\right)(t), \\
\dot{x}_{3}= & \underbrace{-6 x_{1}-2.92 x_{2}-1.2 x_{3}+x_{1}^{2}+d_{3}(t, x)}_{f_{3}(t, x)} \\
& +g_{3}(t, x) \phi_{3}\left(u_{3}\right)(t),
\end{aligned}
$$

where $d_{1}(t, x)=2+2 \sin (2 t), d_{2}(t, x)=3-\cos \left(x_{2}\right), d_{3}(t, x)=$ $0.2+3 \sin (4 t), g_{1}(t, x)=2-\cos (t), g_{2}(t, x)=2-\cos (2 t)$, $g_{3}(t, x)=2-\sin \left(x_{2}\right), m_{i}=2, b_{1 i}=1.5, b_{2 i}=-7, i=1,2,3$.

Firstly, we employ the sliding mode control scheme (see [32]) to control system (43). We assume that the desired trajectory is $x_{d}=\left[x_{1 d}, x_{2 d}, x_{3 d}\right]=[\sin (t), \sin (t), \sin (t)]^{T}$. Let $e_{1}=x_{1}-x_{1 d}, e_{2}=x_{2}-x_{2 d}, e_{3}=x_{3}-x_{3 d}$, and 


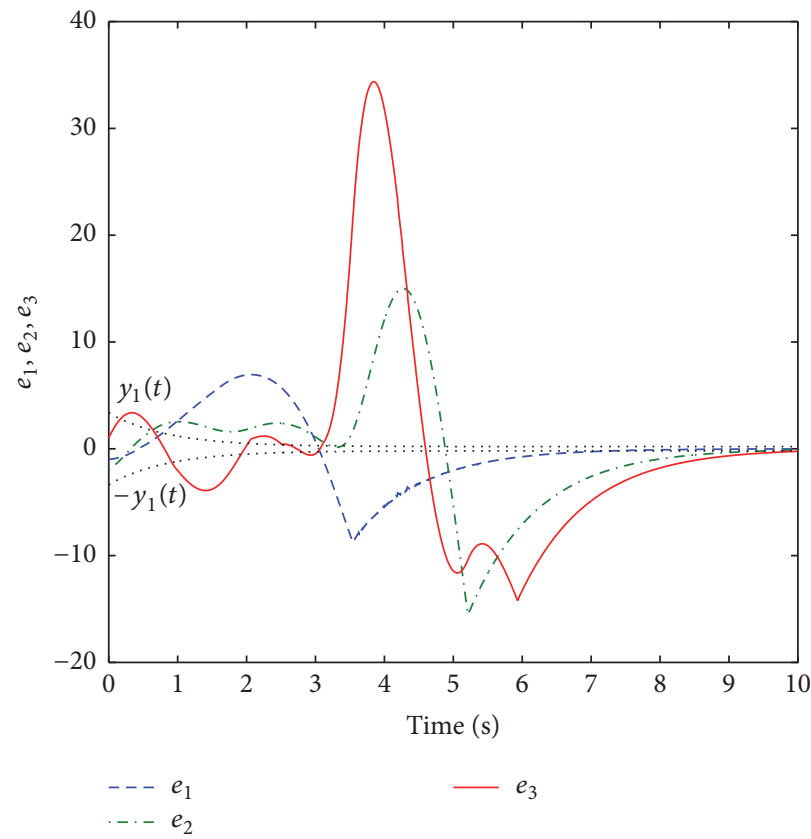

Figure 1: Time responses $e_{1}, e_{2}$, and $e_{3}$ of system (44) by using the control scheme (46).

$\|\operatorname{diag}(g(t, x))\| \geq \delta ; \delta$ is a positive constant. So, the error dynamic system can be rewritten as follows:

$$
\begin{aligned}
& \dot{e}_{1}=f_{1}(t, x)-\dot{x}_{1 d}+g_{1}(t, x) \phi_{1}\left(u_{1}\right)(t), \\
& \dot{e}_{2}=f_{2}(t, x)-\dot{x}_{2 d}+g_{2}(t, x) \phi_{1}\left(u_{2}\right)(t), \\
& \dot{e}_{3}=f_{3}(t, x)-\dot{x}_{3 d}+g_{n}(t, x) \phi_{1}\left(u_{3}\right)(t) .
\end{aligned}
$$

Let $\bar{f}(t, x)=f(t, x)+\operatorname{diag}(g(t, x)) D(u), \operatorname{diag}(\bar{g}(t, x))=$ $\operatorname{diag}(g(t, x)) M$. And the control scheme for error system (24) is designed as

$$
\begin{aligned}
u_{i} & =u_{i 1}+u_{i 2}, \\
u_{i 1} & =-\hat{\bar{g}}_{i}\left(x, \theta_{\bar{g}_{i}}\right)\left[\epsilon+\hat{\bar{g}}_{i}\left(x, \theta_{\bar{g}_{i}}\right)^{2}\right]^{-1}\left[e_{i}-x_{i d}\right. \\
& \left.+\hat{\bar{f}}_{i}\left(x, \theta_{\bar{f}_{i}}\right)+k_{i} \operatorname{sign}\left(s_{i}\right)\right],
\end{aligned}
$$

$$
\begin{aligned}
u_{i 2} & =-\frac{1}{\delta}\left(\varepsilon_{1}+\varepsilon_{2}\left|u_{i 1}\right|+\left[\epsilon+\hat{\bar{g}}_{i}\left(x, \theta_{\bar{g}_{i}}\right)^{2}\right]^{-1}\right. \\
\cdot & {\left.\left[e_{i}-x_{i d}+\hat{\bar{f}}_{i}\left(x, \theta_{\bar{f}_{i}}\right)+k_{i} \operatorname{sign}\left(s_{i}\right)\right]\right) \operatorname{sign}\left(s_{i}\right), } \\
\dot{\theta}_{\bar{f}_{i}} & =\kappa_{\bar{f}_{i}} \psi_{\bar{f}_{i}} s_{i}, \\
\dot{\theta}_{\bar{g}_{i}} & =\kappa_{\bar{g}_{i}} \psi_{\bar{g}_{i}} u_{i 1} s_{i},
\end{aligned}
$$$$
i=1,2,3 \text {, }
$$

where $\epsilon$ is a small positive constant. The initial values of the chaotic system are $\left[x_{1}(0), x_{2}(0), x_{3}(0)\right]^{T}=[-1,-2,1]^{T}$. The design parameters are chosen as follows: $\kappa_{\bar{f}_{i}}=\kappa_{\bar{g}_{i}}=$ $3, i=1,2,3 ., k_{1}=k_{2}=k_{3}=2, \epsilon=0.01, \delta=1$, The initial conditions for the adaptive parameters are selected as $\theta_{\bar{f}_{i}}=\theta_{\bar{g}_{i}}=0.01, i=1,2,3$. By using the sliding mode control scheme (46), the simulation result is shown in Figure 1.

From Figure 1, the error states are beyond the preset boundary $y(t)$ in the previous stage, where $y_{1}(t)=$ $3.17 e^{-1.17 t}+0.2$.

The transformation functions are $s_{i}\left(z_{i}\right)=(2 /$ $\pi) \arctan \left(z_{i}\right), i=1,2,3$. We define three membership functions uniformly distributed on the interval $[-2,2]$. $\delta_{i \text { min }}=\delta_{i \max }=1$. Firstly, according to the proposed control scheme (27), we give a block diagram (see Figure 2).

The simulation result is shown in Figure 3 by using the control scheme (27). In order to improve the control effect, we modify $y_{1}(t)$ as $y_{2}(t)=3.17 e^{-1.17 t}+0.05$. Compared with Figure 3, the tracking errors are improved in Figure 4. From Figure 5, we can see that the chatter phenomenon is eliminated. 


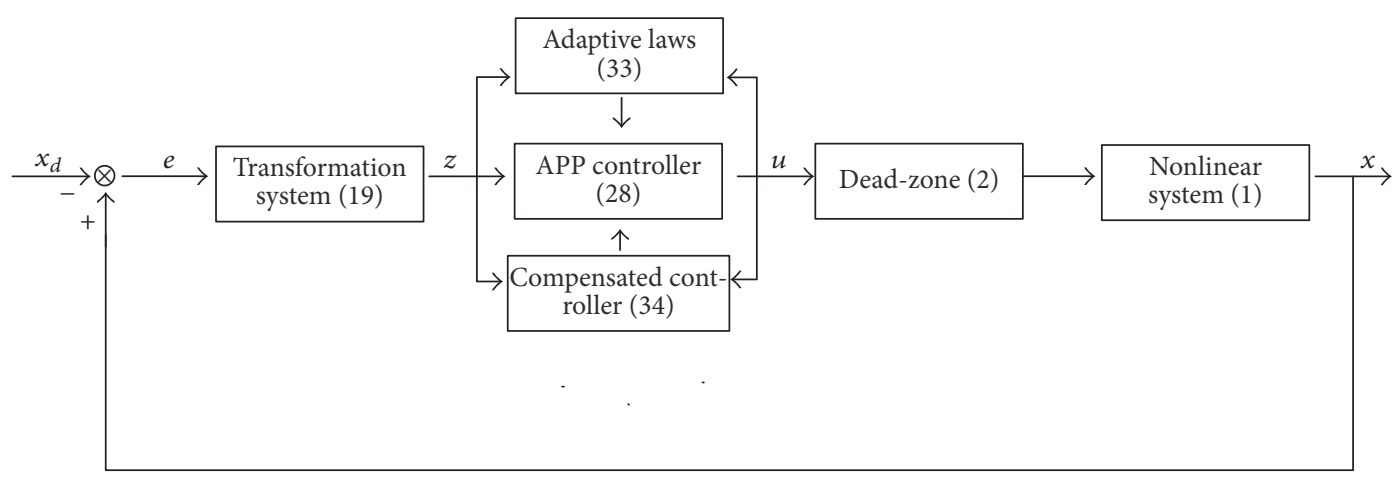

FIGURE 2: A block diagram for the proposed control scheme (27).

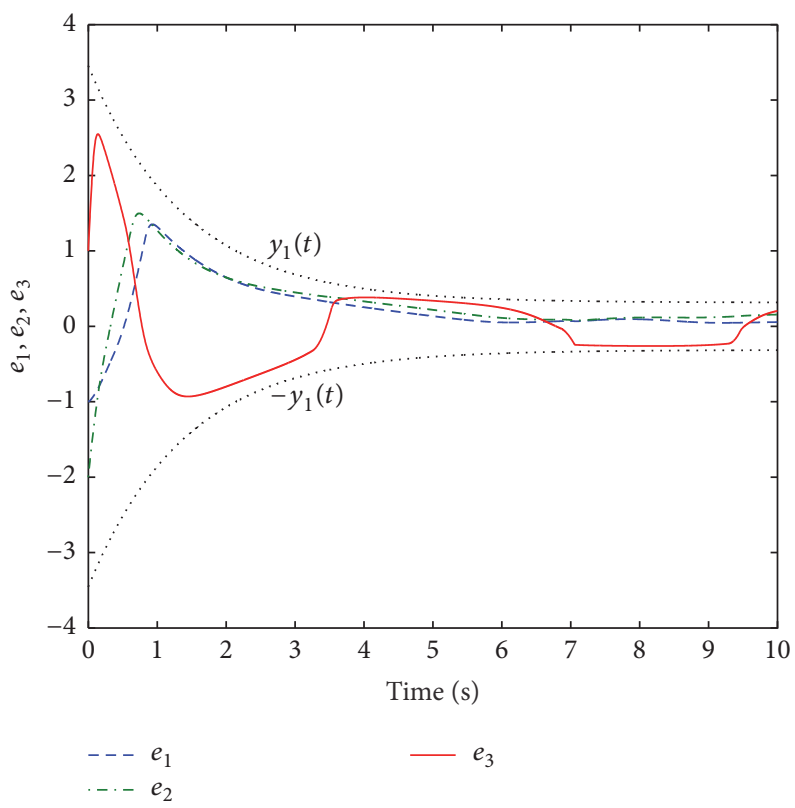

FIGURE 3: Time responses $e_{1}, e_{2}$, and $e_{3}$ of system (44) by using the present control scheme (27) with $y_{1}(t)$.

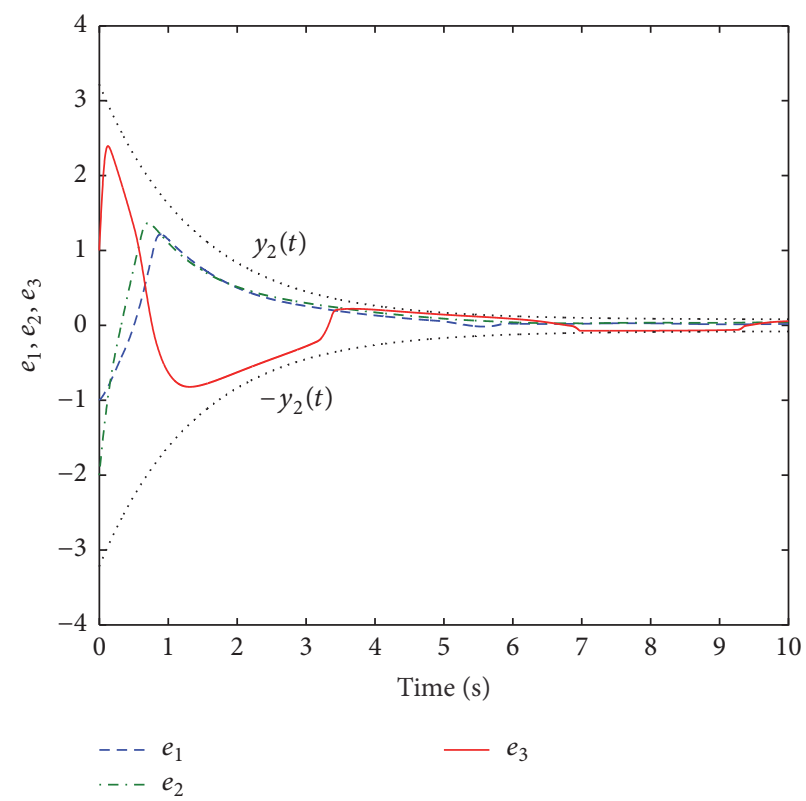

Figure 4: Time responses $e_{1}, e_{2}$, and $e_{3}$ of system (44) by using the present control scheme (27) with $y_{2}(t)$. 


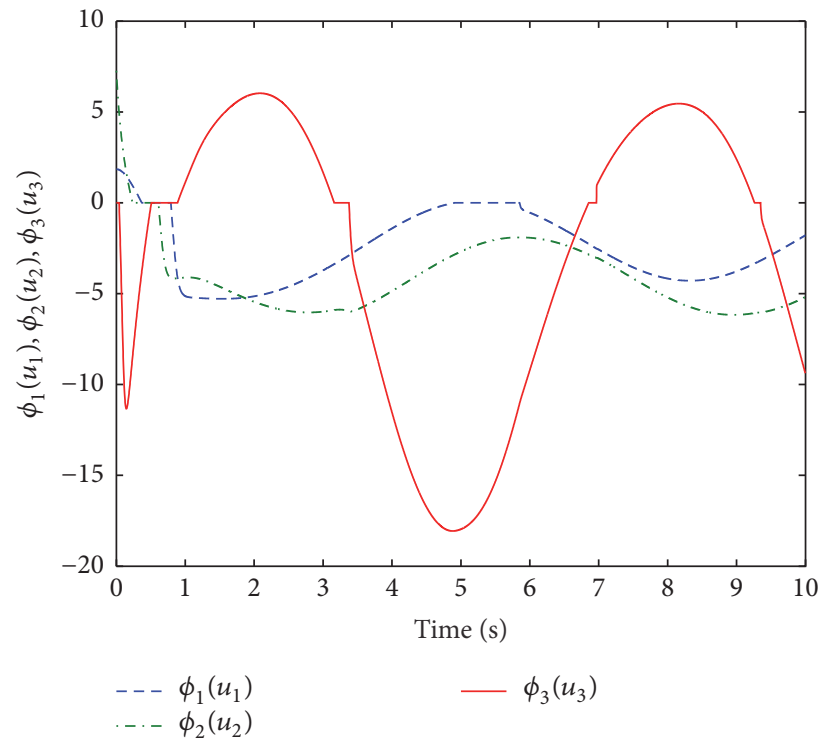

FIGURE 5: Time responses $\phi_{1}\left(u_{1}\right), \phi_{2}\left(u_{2}\right)$, and $\phi_{3}\left(u_{3}\right)$ of system (44) by using the present control scheme (27).

From the simulation results in Figures 4 and 5, we know that the proposed control scheme can guarantee that all the error states are bounded. Moreover, the tracking errors can remain within the prescribed performance bounds all the time without showing chatter phenomenon. So, the proposed control scheme in this paper can achieve the objective.

\section{Conclusions}

In this paper, a fuzzy adaptive prescribed performance control scheme has been developed for a class of uncertain nonlinear systems with unknown control gains and unknown dead-zone inputs. By using fuzzy logic systems and the prescribed performance technique, the stability of the closedloop system has been improved. Simulation results have shown the effectiveness of the proposed scheme.

\section{Conflicts of Interest}

The authors declare that there are no conflicts of interest regarding the publication of this paper.

\section{Acknowledgments}

The authors gratefully acknowledge the support of the National Natural Science Foundation of China (61403157), the Natural Science Foundation of Anhui Province (1508085 QA16), Anhui Province University Humanities and Social Science Research Base project (SK2015A158), the Natural Science Foundation for the Higher Education Institutions of Anhui Province of China (KJ2015A256, KJ2016A666, and KJ2015A178), and the Scientific Research Project of Huainan Normal University (2015xj07zd).

\section{References}

[1] K. T. Sang and G. Li, "Robust nonlinear nominal model following control to over come dead zone nonlinearities," IEEE Transactions on Industrial Electronics, vol. 48, no. 1, pp. 177-184, 2001.

[2] J. Zhou and X. Z. Shen, "Adaptive control of plants with unknown dead-zones," IET Control Theory and Applications, vol. 1, no. 1, pp. 25-32, 2007.

[3] Y.-C. Hung, J.-J. Yan, and T.-L. Liao, "Projective synchronization of Chua's chaotic systems with dead-zone in the control input," Mathematics and Computers in Simulation, vol. 77, no. 4, pp. 374-382, 2008.

[4] A. Boulkroune and M. M'saad, "A practical projective synchronization approach for uncertain chaotic systems with dead-zone input," Communications in Nonlinear Science and Numerical Simulation, vol. 16, no. 11, pp. 4487-4500, 2011.

[5] B. L. Wu, X. B. Cao, and L. Xing, "Robust adaptive control for attitude tracking of spacecraft with unknown dead-zone," Aerospace Science and Technology, vol. 45, pp. 196-202, 2015.

[6] Q. Hu, G. Ma, and L. Xie, "Robust and adaptive variable structure output feedback control of uncertain systems with input nonlinearity," Automatica. A Journal of IFAC, the International Federation of Automatic Control, vol. 44, no. 2, pp. 552-559, 2008.

[7] W. M. Bessa, M. S. Dutra, and E. Kreuzer, "An adaptive fuzzy dead-zone compensation scheme and its application to electrohydraulic systems," Journal of the Brazilian Society of Mechanical Sciences and Engineering, vol. 32, no. 1, pp. 1-7, 2010.

[8] F. L. Lewis, W. K. Tim, L.-Z. Wang, and Z. X. Li, "Deadzone compensation in motion control systems using adaptive fuzzy logic control," IEEE Transactions on Control Systems Technology, vol. 7, no. 6, pp. 731-742, 1999.

[9] K.-K. Shyu, W.-J. Liu, and K.-C. Hsu, "Design of large-scale time-delayed systems with dead-zone input via variable structure control," Automatica, vol. 41, no. 7, pp. 1239-1246, 2005. 
[10] S. Tong, Y. Li, and P. Shi, "Observer-based adaptive fuzzy backstepping output feedback control of uncertain MIMO pure-feedback nonlinear systems," IEEE Transactions on Fuzzy Systems, vol. 20, no. 4, pp. 771-785, 2012.

[11] Y. Li, S. Tong, and T. Li, "Observer-based adaptive fuzzy tracking control of MIMO stochastic nonlinear systems with unknown control directions and unknown dead zones," IEEE Transactions on Fuzzy Systems, vol. 23, no. 4, pp. 1228-1241, 2015.

[12] Y. M. Li, S. C. Tong, Y. J. Liu, and T. S. Li, “Adaptive fuzzy robust output feedback control of nonlinear systems with unknown dead zones based on a small-gain approach," IEEE Transactions on Fuzzy Systems, vol. 22, no. 1, pp. 164-176, 2014.

[13] K.-M. Koo, "Stable adaptive fuzzy controller with time-varying dead-zone," Fuzzy Sets and Systems. An International Journal in Information Science and Engineering, vol. 121, no. 1, pp. 161-168, 2001.

[14] A. Taware and G. Tao, "An adaptive dead-zone inverse controller for systems with sandwiched dead-zones," International Journal of Control, vol. 76, no. 8, pp. 755-769, 2003.

[15] H. Li, C. Wang, P. Shi, and H. Gao, "New passivity results for uncertain discrete-time stochastic neural networks with mixed time delays," Neurocomputing, vol. 73, no. 16-18, pp. 3291-3299, 2010.

[16] D. Meng, "Adaptive neural networks synchronization of a fourdimensional energy resource stochastic system," Abstract and Applied Analysis, vol. 2014, Article ID 863902, 8 pages, 2014.

[17] D. Meng, "Neural networks adaptive synchronization for fourdimension energy resource system with unknown dead zones," Neurocomputing, vol. 151, no. 3, pp. 1495-1499, 2015.

[18] W. Xiang, X. Liu, H. Liu, and Y. Huangfu, "Adaptive fuzzy controller for a class of chaotic system with mismatched uncertainties and unknown control gain matrix," ICIC Express Letters, vol. 7, no. 3 A, pp. 811-817, 2013.

[19] W. Shi, "Adaptive fuzzy control for multi-input multi-output nonlinear systems with unknown dead-zone inputs," Applied Soft Computing Journal, vol. 30, pp. 36-47, 2015.

[20] A. Boulkroune and M. M'Saad, "A fuzzy adaptive variablestructure control scheme for uncertain chaotic MIMO systems with sector nonlinearities and dead-zones," Expert Systems with Applications, vol. 38, no. 12, pp. 14744-14750, 2011.

[21] A. Boulkroune, M. Tadjine, M. M'Saad, and M. Farza, "Fuzzy adaptive controller for MIMO nonlinear systems with known and unknown control direction," Fuzzy Sets and Systems. An International Journal in Information Science and Engineering, vol. 161, no. 6, pp. 797-820, 2010.

[22] E. Kayacan, E. Kayacan, H. Ramon, and W. Saeys, "Adaptive neuro-fuzzy control of a spherical rolling robot using slidingmode-control-theory-based online learning algorithm," IEEE Transactions on Cybernetics, vol. 43, no. 1, pp. 170-179, 2013.

[23] J. Zhou, "Decentralized adaptive control for large-scale timedelay systems with dead-zone input," Automatica. A Journal of IFAC, the International Federation of Automatic Control, vol. 44, no. 7, pp. 1790-1799, 2008.

[24] J. Zhou, C. Wen, and Y. Zhang, "Adaptive output control of nonlinear systems with uncertain dead-zone nonlinearity," Institute of Electrical and Electronics Engineers. Transactions on Automatic Control, vol. 51, no. 3, pp. 504-511, 2006.

[25] X.-S. Wang, C.-Y. Su, and H. Hong, "Robust adaptive control of a class of nonlinear systems with unknown dead-zone," Automatica. A Journal of IFAC, the International Federation of Automatic Control, vol. 40, no. 3, pp. 407-413, 2004.
[26] X. Ye, "Adaptive nonlinear output-feedback control with unknown high-frequency gain sign," Institute of Electrical and Electronics Engineers. Transactions on Automatic Control, vol. 46, no. 1, pp. 112-115, 2001.

[27] C. P. Bechlioulis and G. A. Rovithakis, "Robust adaptive control of feedback linearizable MIMO nonlinear systems with prescribed performance," IEEE Transactions on Automatic Control, vol. 53, no. 9, pp. 2090-2099, 2008.

[28] C. P. Bechlioulis and G. A. Rovithakis, "A low-complexity global approximation-free control scheme with prescribed performance for unknown pure feedback systems," Automatica. A Journal of IFAC, the International Federation of Automatic Control, vol. 50, no. 4, pp. 1217-1226, 2014.

[29] Y. Li and S. Tong, "Prescribed performance adaptive fuzzy output-feedback dynamic surface control for nonlinear largescale systems with time delays," Information Sciences, vol. 292, pp. 125-142, 2015.

[30] Y. M. Li and S. C. Tong, "Adaptive fuzzy output constrained control design for multi-input multioutput stochastic nonstrictfeedback nonlinear systems," IEEE Transactions on Cybernetics, 2016.

[31] Y. Sun and H. Liu, "Fuzzy adaptive prescribed performance control for MIMO uncertain chaotic systems in nonstrict feedback form," Discrete Dynamics in Nature and Society, vol. 2014, Article ID 192387, 6 pages, 2014.

[32] W. Xiang, Y. G. Sun, and H. Liu, "Fuzzy adaptive prescribed performance control for a class of uncertain chaotic systems with unknown control gains," The International Journal of Innovative Computing, Information and Control, vol. 12, no. 2, pp. 603-614, 2016.

[33] J. H. Park, "Synchronization of Genesio chaotic system via backstepping approach," Chaos, Solitons and Fractals, vol. 27, no. 5, pp. 1369-1375, 2006. 


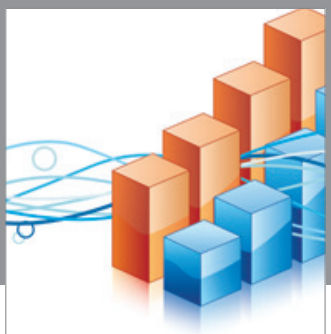

Advances in

Operations Research

vatem alat4

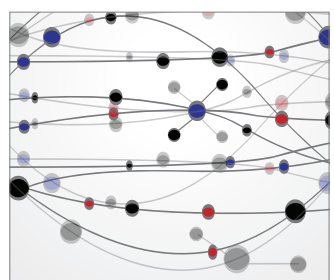

\section{The Scientific} World Journal
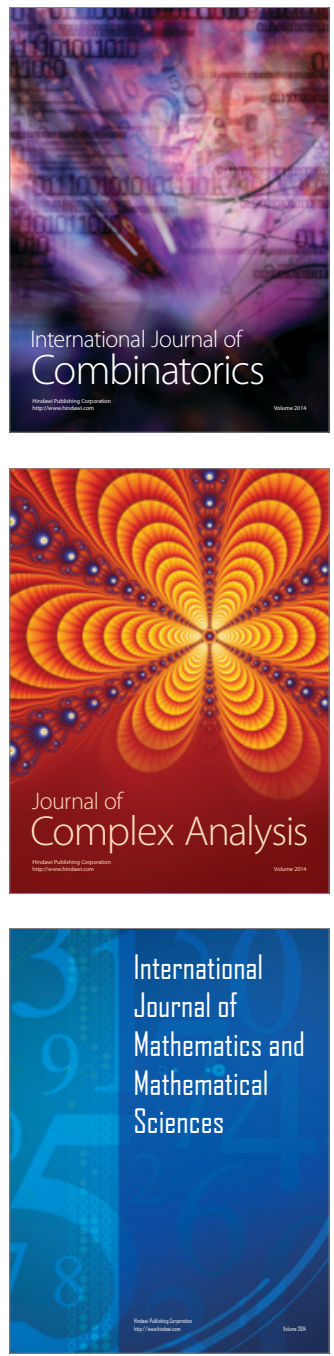
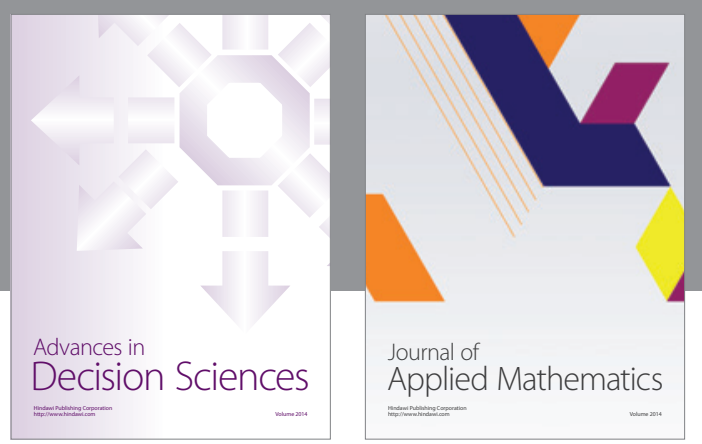

Algebra

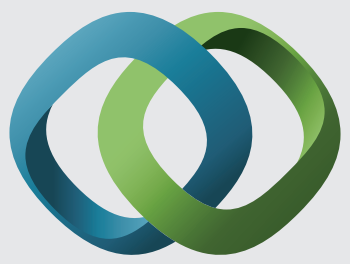

\section{Hindawi}

Submit your manuscripts at

https://www.hindawi.com
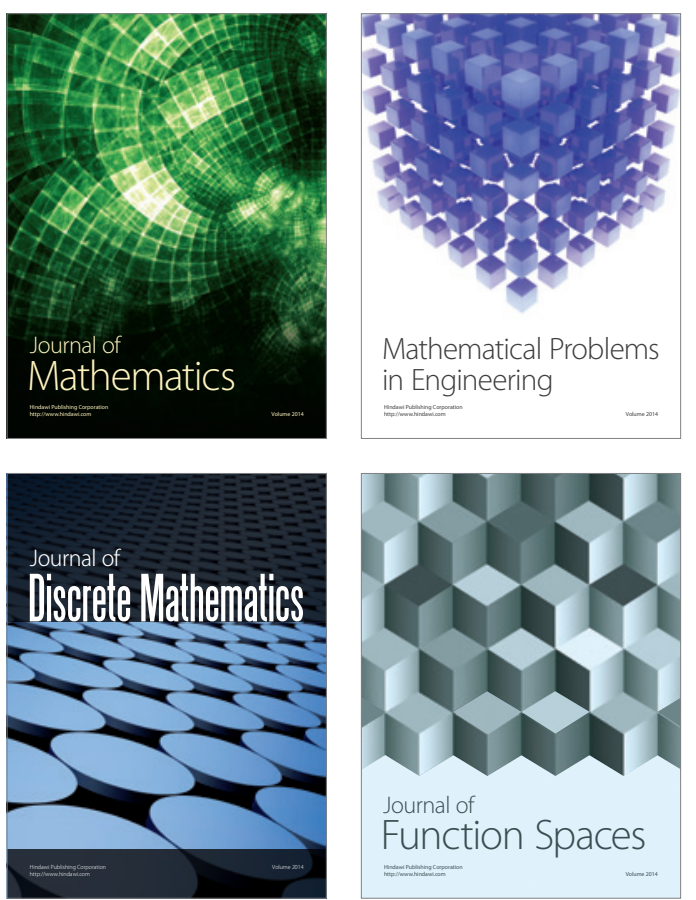

Mathematical Problems in Engineering
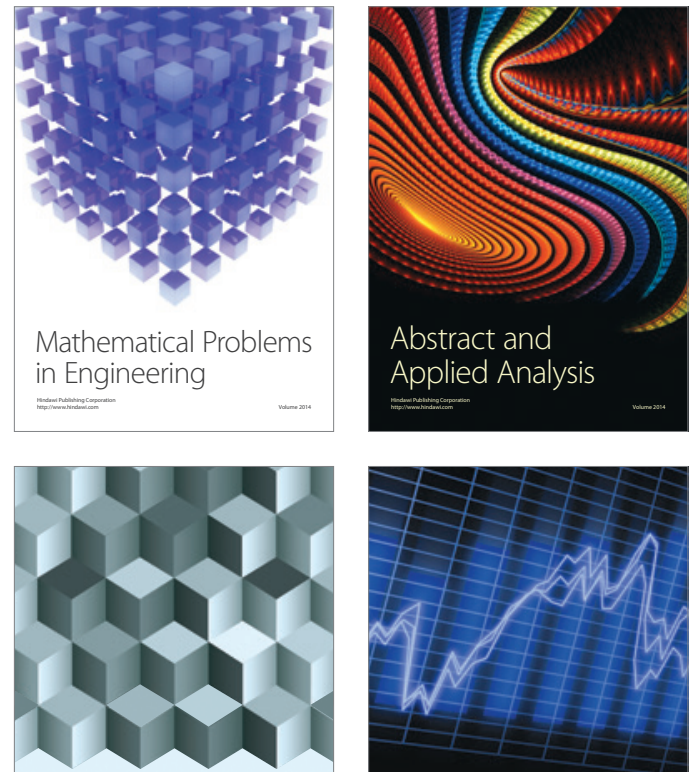

Journal of

Function Spaces

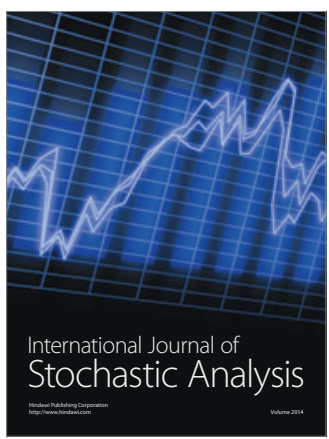

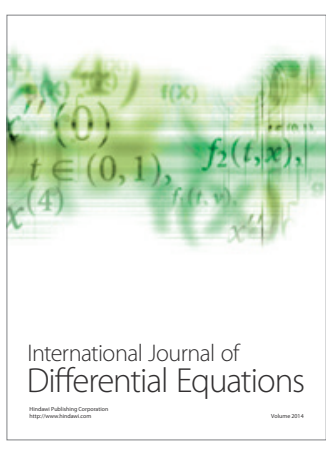
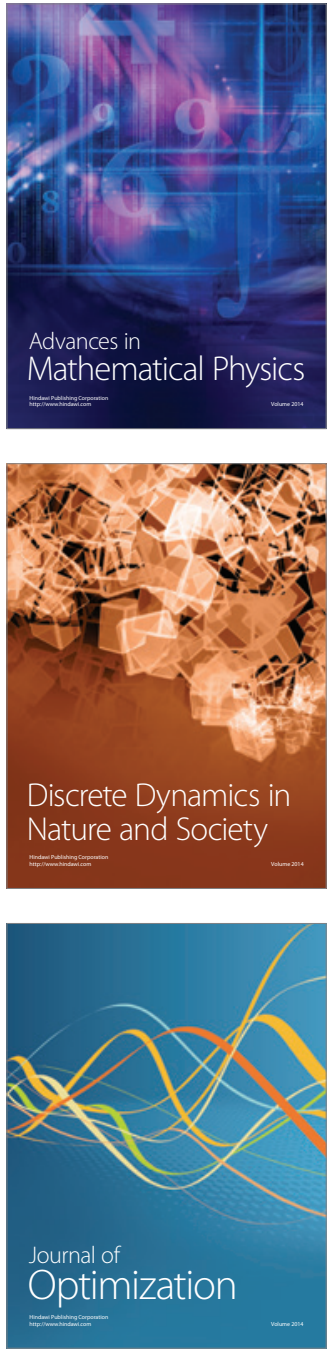